\title{
COCOMMUTATIVE ELEMENTS FORM A MAXIMAL COMMUTATIVE SUBALGEBRA IN QUANTUM MATRICES
}

\author{
SZABOLCS MÉSZÁROS
}

\begin{abstract}
In this paper we prove that the subalgebras of cocommutative elements in the quantized coordinate rings of $M_{n}, G L_{n}$ and $S L_{n}$ are the centralizers of the trace $x_{1,1}+\cdots+x_{n, n}$ in each algebra, for $q \in \mathbb{C}^{\times}$being not a root of unity. In particular, it is not only a commutative subalgebra as it was known before, but it is a maximal one.
\end{abstract}

\section{INTRODUCTION}

In [DL1] M. Domokos and T. Lenagan determined generators for the subalgebra of cocommutative elements in the quantized coordinate ring of the general linear group $\mathcal{O}_{q}\left(G L_{n}(\mathbb{C})\right)$ with $q$ being not a root of unity. Their proof was based on the observation that these are exactly the invariants of some quantum analogue of the conjugation action of $G L_{n}(\mathbb{C})$ on $\mathcal{O}\left(G L_{n}(\mathbb{C})\right)$ which may be called modified adjoint coaction. It turned out that this ring of invariants is basically the same as in the classical setting, namely it is a polynomial ring generated by the quantum versions of the trace functions. In DL2 they proved that it is a more general phenomenon: the subalgebra of cocommutative elements $\mathcal{O}_{q}(G)^{\mathrm{coc}}$ for the quantized coordinate ring $\mathcal{O}_{q}(G)$ of a simply-connected, simple Lie group $G$ is always isomorphic to its classical counterpart $\mathcal{O}(G)^{\mathrm{coc}}$, as a consequence of the Peter-Weyl decomposition for quantized coordinate rings (see $[\mathrm{H}, \mathrm{MNY}]$ ). This way, they obtained generators for the $\mathcal{O}_{q}(G)^{\text {coc }}$ subalgebras and for the related FRT-bialgebras. In the present paper, however, we will discuss a property of $\mathcal{O}_{q}\left(G L_{n}(\mathbb{C})\right)$ that does not hold if $q=1$ or if it is a root of unity.

The correspondence between $\mathcal{O}_{q}(G)^{\mathrm{coc}}$ and $\mathcal{O}(G)^{\mathrm{coc}}$ does not stop on the level of their algebra structure. In the case of $G=G L_{n}(\mathbb{C})$, Aizenbud and Yacobi in [AY] proved the quantum analog of Kostant's theorem stating that $\mathcal{O}_{q}\left(M_{n}(\mathbb{C})\right)$ is a free module over the ring of invariants under the adjoint coaction of $\mathcal{O}_{q}\left(G L_{n}(\mathbb{C})\right)$, provided that $q$ is not a root of unity. Hence, the description of $\mathcal{O}_{q}\left(G L_{n}(\mathbb{C})\right)$ as a module over $\mathcal{O}_{q}\left(G L_{n}(\mathbb{C})\right)^{\text {coc }}$ is available. The classical theorem of Kostant can be interpreted as the $q=1$ case of this result. These type of statements (see [B, JL]) can also be used as tools to obtain other results, as in [Y1 the Joseph localizations being free over certain subalgebras is proved and applied to establish numerous results, including a description of the maximum spectra of $\mathcal{O}_{q}(G)$.

In this paper, we further investigate the relation of the subalgebra $\mathcal{O}_{q}\left(G L_{n}(\mathbb{C})\right)^{\text {coc }}$ to the whole algebra $\mathcal{O}_{q}\left(G L_{n}(\mathbb{C})\right)$ when $q$ is not a root of unity. Namely, we prove the following theorem: 
Theorem 1.1. For $n \in \mathbb{N}^{+}$and $q \in \mathbb{C}^{\times}$not a root of unity, the subalgebra of cocommutative elements is a maximal commutative $\mathbb{C}$-subalgebra in $\mathcal{O}_{q}\left(M_{n}(\mathbb{C})\right)$, $\mathcal{O}_{q}\left(G L_{n}(\mathbb{C})\right)$ and $\mathcal{O}_{q}\left(S L_{n}(\mathbb{C})\right)$.

By Theorem 6.1 in [DL1], these subalgebras are determined by certain pairwise commuting sums of (principal) quantum minors (denoted by $\sigma_{i}, i=1, \ldots, n$ ) that are defined in Section 2. It means that it is enough to prove that the intersection of the centralizers of these explicit commuting generators is not bigger than their generated subalgebra. So we prove the following (stronger) statement:

Theorem 1.2. For $n \in \mathbb{N}^{+}$and $q \in \mathbb{C}^{\times}$not a root of unity, the centralizer of $\sigma_{1}=$ $x_{11}+\cdots+x_{n n}$ in $\mathcal{O}_{q}\left(M_{n}(\mathbb{C})\right)\left(\right.$ resp. $\sigma_{1} \in \mathcal{O}_{q}\left(G L_{n}(\mathbb{C})\right)$ and $\bar{\sigma}_{1} \in \mathcal{O}_{q}\left(S L_{n}(\mathbb{C})\right)$ ) as a unital $\mathbb{C}$-subalgebra is generated by

- $\sigma_{1}, \ldots, \sigma_{n-1}, \sigma_{n}$ in the case of $\mathcal{O}_{q}\left(M_{n}(\mathbb{C})\right)$,

- $\sigma_{1}, \ldots \sigma_{n-1}, \sigma_{n}, \sigma_{n}^{-1}$ in the case of $\mathcal{O}_{q}\left(G L_{n}(\mathbb{C})\right)$, and

- $\bar{\sigma}_{1}, \ldots \bar{\sigma}_{n-1}$ in the case of $\mathcal{O}_{q}\left(S L_{n}(\mathbb{C})\right)$.

It is important to note that, while the theorems in [DL1, DL2] are quantum analogues of theorems established in the commutative case and they are also true if $q$ is a root of unity (see $[\mathrm{AZ}]$ ), this result, however, has no direct commutative counterpart and also fails if $q$ is a root of unity since then the algebras have large center.

In Ore extensions of polynomials rings or in lower Gelfand-Kirillov dimension, it is not a rare phenomenon that a centralizer of an element $a \in A$ is commutative but larger than $\mathbb{C}\langle a, Z(A)\rangle$, see $\mathrm{BS}, \mathrm{RS}$. The above investigation shows that it also occurs in less regular situations for some very special elements in quantized function algebras.

As a consequence of Theorem 1.1, we can get other maximal commutative subalgebras by applying automorphisms. One of these automorphic images is the invariants of the adjoint coaction, as it is discussed in Remark 5.2. Moreover, by an analogous argument as we use in the proof of Theorem 1.1. it is possible to find maximal Poisson-commutative subalgebras in the semi-classical limits. We will discuss these issues in a subsequent paper.

The article is organized as follows: In the next section we introduce the relevant notions and notations. In Section [3, first we prove Proposition 3.1 stating that it is enough to prove Theorem 1.2 for any of the three algebras $\mathcal{O}_{q}\left(M_{n}(\mathbb{C})\right)$, $\mathcal{O}_{q}\left(G L_{n}(\mathbb{C})\right)$ or $\mathcal{O}_{q}\left(S L_{n}(\mathbb{C})\right)$. Then, in Section 4 we discuss the proof of case $n=2$ as a starting step of the induction used to prove Theorem 1.2. Finally, in Section 5 we prove the induction step to complete the proof of the theorem.

\section{Preliminaries}

2.1. Quantized coordinate rings. Assume that $n \in \mathbb{N}^{+}$and $q \in \mathbb{C}^{\times}$is not a root of unity. Define $\mathcal{O}_{q}\left(M_{n}(\mathbb{C})\right)$, the quantized coordinate ring of $n \times n$ matrices as the unital $\mathbb{C}$-algebra generated by the $n^{2}$ generators $x_{i, j}$ for $1 \leq i, j \leq n$ that are subject to the following relations:

$$
x_{i, j} x_{k, l}= \begin{cases}x_{k, l} x_{i, j}+\left(q-q^{-1}\right) x_{i, l} x_{k, j} & \text { if } i<k \text { and } j<l \\ q x_{k, l} x_{i, j} & \text { if }(i=k \text { and } j<l) \text { or }(j=l \text { and } i<k) \\ x_{k, l} x_{i, j} & \text { otherwise }\end{cases}
$$


for all $1 \leq i, j, k, l \leq n$. It turns out to be a finitely generated $\mathbb{C}$-algebra which is a Noetherian domain. (For a detailed exposition, see [BG].) Furthermore, it can be endowed with a coalgebra structure by setting $\varepsilon\left(x_{i, j}\right)=\delta_{i, j}$ and $\Delta\left(x_{i, j}\right)=$ $\sum_{k=1}^{n} x_{i, k} \otimes x_{k, j}$ turning $\mathcal{O}_{q}\left(M_{n}(\mathbb{C})\right)$ into a bialgebra.

Similarly, one can define the non-commutative deformations of the coordinate rings of $G L_{n}$ and $S L_{n}$ using the quantum determinant

$$
\operatorname{det}_{q}:=\sum_{s \in S_{n}}(-q)^{\ell(s)} x_{1, s(1)} x_{2, s(2)} \ldots x_{n, s(n)}
$$

where $\ell(\sigma)$ stands for the length of $\sigma$ in the Coxeter group $S_{n}$. This definition can be "legitimized" by considering the quantum exterior algebra $\Lambda_{q}\left(\mathbb{C}^{n}\right)$ (see $\mathrm{BG}$ ). Also its special behavior is justified by the fact that it is a group-like element (i.e. $\left.\Delta\left(\operatorname{det}_{q}\right)=\operatorname{det}_{q} \otimes \operatorname{det}_{q}\right)$ and it generates the center of $\mathcal{O}_{q}\left(M_{n}(\mathbb{C})\right)$. Then analogously to the classical case - one defines

$$
\mathcal{O}_{q}\left(S L_{n}(\mathbb{C})\right):=\mathcal{O}_{q}\left(M_{n}(\mathbb{C})\right) /\left(\operatorname{det}_{q}-1\right) \quad \mathcal{O}_{q}\left(G L_{n}(\mathbb{C})\right):=\mathcal{O}_{q}\left(M_{n}(\mathbb{C})\right)\left[\operatorname{det}_{q}^{-1}\right]
$$

where inverting $\operatorname{det}_{q}$ cannot cause any problem because it is central hence normal. The comultiplication and counit on $\mathcal{O}_{q}\left(M_{n}(\mathbb{C})\right)$ induce coalgebra structures on these algebras as well. In particular, $\mathcal{O}_{q}\left(M_{n}(\mathbb{C})\right)$ is a subbialgebra of $\mathcal{O}_{q}\left(G L_{n}(\mathbb{C})\right)$. In the case of $\mathcal{O}_{q}\left(S L_{n}(\mathbb{C})\right)$ and $\mathcal{O}_{q}\left(G L_{n}(\mathbb{C})\right)$ it is possible to define antipodes that turn them into Hopf algebras.

2.2. Quantum minors. We call an element $a$ of a coalgebra $A$ cocommutative if $\Delta(a)=(\tau \circ \Delta)(a)$ where $\tau: A \otimes A \rightarrow A \otimes A$ is the flip $\tau(a \otimes b)=b \otimes a$. Hence, we can define $A^{\text {coc }}$, the subset of cocommutative elements in $A$ which is necessarily a subalgebra if $A$ is a bialgebra. For $A=\mathcal{O}_{q}\left(M_{n}(\mathbb{C})\right)$ the quantum determinant is cocommutative since it is group-like. Moreover, by generalizing the notion of $\operatorname{det}_{q}$, one can give an explicit description of $A^{\mathrm{coc}}$ as it is proved in [DL1]. For this purpose, let us define the quantum minors for $I, J \subseteq\{1, \ldots, n\}, I=\left(i_{1}, \ldots, i_{t}\right)$ and $J=\left(j_{1}, \ldots, j_{t}\right)$ as

$$
[I \mid J]:=\sum_{s \in S_{t}}(-q)^{\ell(s)} x_{i_{1}, j_{s(1)}} \ldots x_{i_{t}, j_{s(t)}}=\operatorname{det}_{q}\left(\mathbb{C}\left\langle x_{i, j} \mid i \in I, j \in J\right\rangle\right) \in A
$$

where $\mathbb{C}\langle\ldots\rangle$ stands for the generated $\mathbb{C}$-subalgebra and $\operatorname{det}_{q}\left(\mathbb{C}\left\langle x_{i, j} \mid i \in I, j \in J\right\rangle\right)$ denotes the quantum determinant of the subalgebra generated by $\left\{x_{i, j}\right\}_{i \in I, j \in J}$, which can be identified with $\mathcal{O}_{q}\left(M_{t}(\mathbb{C})\right)$. Now, one may compute

$$
\Delta([I \mid J])=\sum_{|K|=t}[I \mid K] \otimes[K \mid J]
$$

so we get cocommutative elements by taking

$$
\sigma_{i}=\sum_{|I|=i}[I \mid I] \in \mathcal{O}_{q}\left(M_{n}(\mathbb{C})\right)
$$

for all $1 \leq i \leq n$. For $i=n$ we get $\operatorname{det}_{q}$ again and in the case of $i=1$, it is $\sigma_{1}=x_{1,1}+x_{2,2}+\cdots+x_{n, n}$.

We will use $\sigma_{i}$ and $\bar{\sigma}_{i}$ for the induced elements

$$
\bar{\sigma}_{i}=\sigma_{i}+\left(\operatorname{det}_{q}-1\right) \in \mathcal{O}_{q}\left(S L_{n}(\mathbb{C})\right) \quad \sigma_{i} \in \mathcal{O}_{q}\left(M_{n}(\mathbb{C})\right) \leq \mathcal{O}_{q}\left(G L_{n}(\mathbb{C})\right)
$$

an we will write $\sigma_{i}(A)$ for $\sigma_{i}$ in an algebra $A$ isomorphic to $\mathcal{O}_{q}\left(M_{t}(\mathbb{C})\right)$ for some $t$. Theorem 6.1 in DL1 states that the subalgebra of cocommutative elements 
in $\mathcal{O}_{q}\left(M_{n}(\mathbb{C})\right)$ is freely generated (as a commutative algebra) by $\sigma_{1}, \ldots, \sigma_{n}$, and (consequently) in $\mathcal{O}_{q}\left(G L_{n}(\mathbb{C})\right)$ it is generated by $\sigma_{1}, \ldots, \sigma_{n}, \sigma_{n}^{-1}$, giving an algebra isomorphic to $\mathbb{C}\left[t_{1}, \ldots, t_{n}, t_{n}^{-1}\right]$. The case of $S L_{n}$ is proved in DL2]: $\mathcal{O}_{q}\left(S L_{n}(\mathbb{C})\right)^{\text {coc }}$ is generated by $\bar{\sigma}_{1}, \ldots, \bar{\sigma}_{n-1}$ and is isomorphic to $\mathbb{C}\left[t_{1}, \ldots, t_{n-1}\right]$.

2.3. Poincaré-Birkhoff-Witt basis in the quantized coordinate ring of matrices. Several properties of $\mathcal{O}_{q}\left(M_{n}(\mathbb{C})\right)$ can be deduced by the observation that it is an iterated Ore extension. It means that there exists a finite sequence of $\mathbb{C}$-algebras $R_{0}, R_{1}, \ldots, R_{n^{2}}$ such that $R_{0}=\mathbb{C}$ and $R_{i+1}=R_{i}\left[x_{i} ; \tau_{i}, \delta_{i}\right]$, the skew polynomial ring in $x_{i}$ for an appropriate automorphism $\tau_{i} \in \operatorname{Aut}\left(R_{i}\right)$ and a derivation $\delta_{i} \in \operatorname{Der}\left(R_{i}\right)$.

This choice of sequence of subalgebras includes an ordering on the variables that is - from the several possible options, now - the lexicographic ordering on $\{1, \ldots, n\} \times\{1, \ldots, n\}$. Moreover, an iterated Ore extension as $\mathcal{O}_{q}\left(M_{n}(\mathbb{C})\right)$ has a Poincaré-Birkhoff-Witt basis, i.e. a $\mathbb{C}$-basis consisting of the ordered monomials of the variables $x_{i, j}$. So, in the following, we will refer to the following basis as the monomial basis of $\mathcal{O}_{q}\left(M_{n}(\mathbb{C})\right)$ :

$$
x_{1,1}^{k_{1,1}} x_{1,2}^{k_{1,2}} x_{1,3}^{k_{1,3}} \ldots x_{1, n}^{k_{1, n}} x_{2,1}^{k_{2,1}} \ldots x_{n, n}^{k_{n, n}} \quad\left(k_{i j} \in \mathbb{N}, i, j \in\{1,2, \ldots, n\}\right)
$$

It is indeed a basis, see $\mathrm{BG}$.

Since the defining relations of $\mathcal{O}_{q}\left(M_{n}(\mathbb{C})\right)$ are homogeneous with respect to the total degree in the free algebra, $\mathcal{O}_{q}\left(M_{n}(\mathbb{C})\right)$ inherits an $\mathbb{N}$-graded algebra structure, i.e.

$$
\mathcal{O}_{q}\left(M_{n}(\mathbb{C})\right)=\bigoplus_{d \in \mathbb{N}} \mathcal{O}_{q}\left(M_{n}(\mathbb{C})\right)_{d}
$$

as a vector space and $\mathcal{O}_{q}\left(M_{n}(\mathbb{C})\right)_{d} \cdot \mathcal{O}_{q}\left(M_{n}(\mathbb{C})\right)_{e} \subseteq \mathcal{O}_{q}\left(M_{n}(\mathbb{C})\right)_{d+e}$ for all $d, e \in \mathbb{N}$. Consequently, we may define a degree function $\operatorname{deg}: \mathcal{O}_{q}\left(M_{n}(\mathbb{C})\right) \rightarrow \mathbb{N}$ as the maximum of the degrees of nonzero homogeneous components.

Although $\operatorname{det}_{q}-1$ is not homogeneous with respect to the total degree, it is homogeneous modulo $n$ so the quotient algebra $\mathcal{O}_{q}\left(S L_{n}(\mathbb{C})\right)$ becomes a $\mathbb{Z} / n \mathbb{Z}$ graded algebra.

2.4. Associated graded ring. For a filtered $\operatorname{ring}\left(R,\left\{\mathcal{F}^{d}\right\}_{d \in \mathbb{N}}\right)$ i.e. where $\left\{\mathcal{F}^{d}\right\}_{d \in \mathbb{N}}$ is an ascending chain of subspaces in $R$ such that $R=\cup_{d \in \mathbb{N}} \mathcal{F}^{d}$ and $\mathcal{F}^{d} \cdot \mathcal{F}^{e} \subseteq \mathcal{F}^{d+e}$ for all $d, e \in \mathbb{N}$, we define its associated graded ring

$$
\operatorname{gr}(R):=\bigoplus_{d \in \mathbb{N}} \mathcal{F}^{d} / \mathcal{F}^{d-1}
$$

where we use the notation $\mathcal{F}^{-1}=\{0\}$. The multiplication of $\operatorname{gr}(R)$ is defined in the usual way:

$$
\begin{gathered}
\mathcal{F}^{d} / \mathcal{F}^{d-1} \times \mathcal{F}^{e} / \mathcal{F}^{e-1} \rightarrow \mathcal{F}^{d+e} / \mathcal{F}^{d+e-1} \\
\left(x+\mathcal{F}^{d-1}, y+\mathcal{F}^{e-1}\right) \mapsto x y+\mathcal{F}^{d+e-1}
\end{gathered}
$$

Clearly, it is a graded algebra by definition. In fact, $\operatorname{gr}($.$) can be made into a$ functor defined as follows: for a morphism of filtered algebras $f:\left(R,\left\{\mathcal{F}^{d}\right\}_{d \in \mathbb{N}}\right) \rightarrow$ $\left(S,\left\{\mathcal{G}^{d}\right\}_{d \in \mathbb{N}}\right)$ (i.e. when $\left.f\left(\mathcal{F}^{d}\right) \subseteq \mathcal{G}^{d}\right)$ we define

$$
\begin{gathered}
\operatorname{gr}(f): \operatorname{gr}(R) \rightarrow \operatorname{gr}(S) \\
\left(x_{d}+\mathcal{F}^{d-1}\right)_{d \in \mathbb{N}} \mapsto\left(f\left(x_{d}\right)+\mathcal{G}^{d-1}\right)_{d \in \mathbb{N}}
\end{gathered}
$$


One can check that it is indeed well defined and preserves composition. A basic property of $\operatorname{gr}($.$) is that if we have a map f: R \rightarrow S$ such that $f\left(\mathcal{F}^{d}\right)=\mathcal{G}^{d}$ then the $\operatorname{gr}(f)$ is also surjective.

\section{EquivalenCE OF THE STATEMENTS}

As it is mentioned in the introduction, Theorem 1.1 follows directly from Theorem 1.2. Indeed, since $\sigma_{i}$ 's are commuting generators in the subalgebra of cocommutative elements in $\mathcal{O}_{q}\left(M_{n}(\mathbb{C})\right), \mathcal{O}_{q}\left(G L_{n}(\mathbb{C})\right)$ and $\mathcal{O}_{q}\left(S L_{n}(\mathbb{C})\right.$ ) (see Section 2$)$, any commutative subalgebra containing the subalgebra of cocommutative elements is contained in the centralizer of $\sigma_{1}$.

Moreover, the following proposition shows that it is enough to prove Theorem 1.2 in the case of $\mathcal{O}_{q}\left(M_{n}(\mathbb{C})\right)$.

Proposition 3.1. Assume that $n \in \mathbb{N}^{+}$and $q \in \mathbb{C}^{\times}$is not a root of unity. The following are equivalent:

(1) The centralizer of $\sigma_{1} \in \mathcal{O}_{q}\left(M_{n}(\mathbb{C})\right)$ is generated by $\sigma_{1}, \ldots, \sigma_{n-1}, \sigma_{n}$.

(2) The centralizer of $\sigma_{1} \in \mathcal{O}_{q}\left(G L_{n}(\mathbb{C})\right)$ is generated by $\sigma_{1}, \ldots, \sigma_{n-1}, \sigma_{n}, \sigma_{n}^{-1}$.

(3) The centralizer of $\bar{\sigma}_{1} \in \mathcal{O}_{q}\left(S L_{n}(\mathbb{C})\right)$ is generated by $\bar{\sigma}_{1}, \ldots, \bar{\sigma}_{n-1}$.

For the proof, we need the following short lemma:

Lemma 3.2. Let $R=\oplus_{i \geq 0} R_{i}$ be an $\mathbb{N}$-graded algebra and $r \in R_{k}$ a central element that is not a zero-divisor. Then for all $d \in \mathbb{N},(r-1) \cap R_{d}=0$.

Proof. Since $r-1$ is central, its generated ideal is its generated left ideal so $0 \neq x \in$ $(r-1)$ means that $x=y \cdot(r-1)$ for some $y \in R$. Let $y=\sum_{i=i_{0}}^{\operatorname{deg} y} y_{i} \in \oplus_{i} R_{i}$ be the homogeneous decomposition of $y$ where $y_{i_{0}} \neq 0$. Then the highest degree nonzero homogeneous component of $y \cdot(r-1)$ is $y_{\operatorname{deg} y} r$ which is of degree deg $y+k$ since $r$ is not a zero-divisor. While the lowest degree nonzero component of $y \cdot(r-1)$ is $-y_{i_{0}}$ which is of degree $i_{0} \leq \operatorname{deg} y<\operatorname{deg} y+k$. Therefore, $x$ cannot be homogeneous.

Proof of Proposition 3.1. Assume that 1) is true and let $h \in \mathcal{O}_{q}\left(G L_{n}(\mathbb{C})\right)$ that commutes with $\sigma_{1}$. By the definition of $\mathcal{O}_{q}\left(G L_{n}(\mathbb{C})\right)$, there exists an $k \in \mathbb{N}$ such that $h \cdot \operatorname{det}_{q}^{k} \in \mathcal{O}_{q}\left(M_{n}(\mathbb{C})\right) \leq \mathcal{O}_{q}\left(G L_{n}(\mathbb{C})\right)$ which also commutes with $\sigma_{1}$ since $\operatorname{det}_{q}$ is central. Therefore, by 1) we have $h \cdot \operatorname{det}_{q}^{k} \in \mathbb{C}\left\langle\sigma_{1}, \ldots, \sigma_{n-1}, \sigma_{n}\right\rangle$ hence $h=h \cdot \operatorname{det}_{q}^{k}$. $\operatorname{det}_{q}^{-k} \in \mathbb{C}\left\langle\sigma_{1}, \ldots, \sigma_{n-1}, \sigma_{n}, \sigma_{n}^{-1}\right\rangle$ and so 2) follows. Conversely, assume 2) and take an $h \in \mathcal{O}_{q}\left(M_{n}(\mathbb{C})\right) \subseteq \mathcal{O}_{q}\left(G L_{n}(\mathbb{C})\right)$ that commutes with $\sigma_{1}$. By the assumption, $h \in \mathbb{C}\left\langle\sigma_{1}, \ldots, \sigma_{n-1}, \sigma_{n}, \sigma_{n}^{-1}\right\rangle$ hence it is cocommutative in $\mathcal{O}_{q}\left(G L_{n}(\mathbb{C})\right)$. Since $\mathcal{O}_{q}\left(M_{n}(\mathbb{C})\right)$ is a subbialgebra of $\mathcal{O}_{q}\left(G L_{n}(\mathbb{C})\right), h$ is cocommutative in $\mathcal{O}_{q}\left(M_{n}(\mathbb{C})\right)$ too, hence by $\mathcal{O}_{q}\left(M_{n}(\mathbb{C})\right)^{\text {coc }}=\mathbb{C}\left\langle\sigma_{1}, \ldots, \sigma_{n-1}, \sigma_{n}\right\rangle$ (see Section 2) 1) follows.

Now, we prove 1) $\Longleftrightarrow 3)$ : First, assume 1) and let $\bar{h} \in \mathcal{O}_{q}\left(S L_{n}(\mathbb{C})\right)$ that commutes with $\bar{\sigma}_{1}$. Since $\mathcal{O}_{q}\left(S L_{n}(\mathbb{C})\right)$ is $\mathbb{Z} / n \mathbb{Z}$-graded and $\bar{\sigma}_{1}$ is homogeneous with respect to this grading, its centralizer is generated by homogeneous elements. So we may assume that $\bar{h}$ is homogeneous as well. Let $k=\operatorname{deg}(\bar{h})$. Take an $h \in$ $\mathcal{O}_{q}\left(M_{n}(\mathbb{C})\right)$ that represents $\bar{h} \in \mathcal{O}_{q}\left(S L_{n}(\mathbb{C})\right)=\mathcal{O}_{q}\left(M_{n}(\mathbb{C})\right) /\left(\operatorname{det}_{q}-1\right)$. Let $h=$ $\sum_{j=0}^{d} h_{j n+k}$ be the $\mathbb{N}$-homogeneous decomposition of $h$ where $h_{j n+k}$ is homogeneous of degree $j n+k$ for all $j \in \mathbb{N}$. (We do not need the other homogeneous components as $\bar{h}$ is $\mathbb{Z} / n \mathbb{Z}$-homogeneous so we may assume that $h$ has nonzero homogeneous 
components only in degrees $\equiv \operatorname{deg}(\bar{h})$ modulo $n$.) Then we can take

$$
h^{\prime}:=\sum_{j=0}^{d} h_{j n+k} \cdot \operatorname{det}_{q}^{d-j} \in \mathcal{O}_{q}\left(M_{n}(\mathbb{C})\right)_{d n+k}
$$

which is a homogeneous element of degree $d n+k$ representing $\bar{h}$ in $\mathcal{O}_{q}\left(M_{n}(\mathbb{C})\right)$. Therefore, $\sigma_{1} h^{\prime}-h^{\prime} \sigma_{1} \in\left(\operatorname{det}_{q}-1\right) \cap \mathcal{O}_{q}\left(M_{n}(\mathbb{C})\right)_{d n+k+1}$ because $\bar{\sigma}_{1} \bar{h}-\bar{h} \bar{\sigma}_{1}=$ $0 \in \mathcal{O}_{q}\left(S L_{n}(\mathbb{C})\right)$ and $\sigma_{1}$ is homogeneous of degree 1. By Lemma 3.2, we get $\left(\operatorname{det}_{q}-1\right) \cap \mathcal{O}_{q}\left(M_{n}(\mathbb{C})\right)_{d n+k+1}=0$ meaning $\sigma_{1} h^{\prime}=h^{\prime} \sigma_{1}$. Then applying 1$)$ gives $h^{\prime} \in \mathbb{C}\left\langle\sigma_{1}, \ldots, \sigma_{n}\right\rangle$ hence $\bar{h} \in \mathbb{C}\left\langle\overline{\sigma_{1}}, \ldots, \overline{\sigma_{n-1}}\right\rangle$ as we claimed.

Conversely, assume 3) and let $h \in \mathcal{O}_{q}\left(M_{n}(\mathbb{C})\right)$ such that $\sigma_{1} h=h \sigma_{1}$. Since $\sigma_{1}$ is $\mathbb{N}$-homogeneous, its centralizer is also generated by homogeneous elements so we may assume that $h$ is homogeneous. Then we can take the image $\bar{h}$ of $h$ in $\mathcal{O}_{q}\left(S L_{n}(\mathbb{C})\right)$ which is homogeneous with respect to the $\mathbb{Z} / n \mathbb{Z}$-grading of $\mathcal{O}_{q}\left(S L_{n}(\mathbb{C})\right)$. Let $k=\operatorname{deg}(\bar{h})$. By the assumption, $\bar{h}$ commutes with $\bar{\sigma}_{1}$ hence $\bar{h} \in \mathbb{C}\left\langle\bar{\sigma}_{1}, \ldots, \bar{\sigma}_{n-1}\right\rangle$ by 3$)$. This decomposition of $\bar{h}$ can be lifted to $\mathcal{O}_{q}\left(M_{n}(\mathbb{C})\right)$ giving an element $s \in \mathbb{C}\left\langle\sigma_{1}, \ldots, \sigma_{n-1}\right\rangle$ such that $h-s \in\left(\operatorname{det}_{q}-1\right)$. As $\bar{h}$ was $\mathbb{Z} / n \mathbb{Z}$ homogeneous, $s$ can also be chosen to be $\mathbb{Z} / n \mathbb{Z}$-homogeneous since $\bar{\sigma}_{1}, \ldots, \bar{\sigma}_{n-1}$ are $\mathbb{Z} / n \mathbb{Z}$-homogeneous. Let $d=\frac{1}{n}(\max (\operatorname{deg} h, \operatorname{deg} s)-k)$ and take $s=\sum_{j=0}^{d} s_{j n+k}$ the homogeneous decomposition of $s$. If $\operatorname{deg}(s)>\operatorname{deg}(h)$ then let

$$
h^{\prime}=h \cdot \operatorname{det}_{q}^{\frac{1}{n}}(\operatorname{deg}(s)-\operatorname{deg}(h))
$$

so now $d=\operatorname{deg}(s)=\operatorname{deg}\left(h^{\prime}\right)$. (The exponent is an integer since $\operatorname{deg}(h)=\operatorname{deg}(s)$ modulo $n$.) Otherwise, let $h^{\prime}=h$.

Now, the same way as in the proof of 1$) \Rightarrow 3$ ), we can modify $s$ as follows: Let

$$
s^{\prime}:=\sum_{j=0}^{d} s_{j n+k} \cdot \operatorname{det}_{q}^{d-j}
$$

Then $s^{\prime} \in \mathbb{C}\left\langle\sigma_{1}, \ldots, \sigma_{n}\right\rangle$, it is $\mathbb{N}$-homogeneous of degree $n d+k$, and $s-s^{\prime} \in$ $\left(\operatorname{det}_{q}-1\right)$. So $h^{\prime}-s^{\prime}=\left(h^{\prime}-h\right)+(h-s)+\left(s-s^{\prime}\right) \in\left(\operatorname{det}_{q}-1\right) \cap \mathcal{O}_{q}\left(M_{n}(\mathbb{C})\right)_{n d+k}$ which is zero by Lemma 3.2. Hence, $h^{\prime} \in \mathbb{C}\left\langle\sigma_{1}, \ldots, \sigma_{n}\right\rangle$ which gives $h \in \mathbb{C}\left\langle\sigma_{1}, \ldots, \sigma_{n}, \sigma_{n}^{-1}\right\rangle$. However, $\mathbb{C}\left\langle\sigma_{1}, \ldots, \sigma_{n}, \sigma_{n}^{-1}\right\rangle \cap \mathcal{O}_{q}\left(M_{n}(\mathbb{C})\right)=\mathbb{C}\left\langle\sigma_{1}, \ldots, \sigma_{n}\right\rangle$ as they are the subalgebras of cocommutative elements in $\mathcal{O}_{q}\left(G L_{n}(\mathbb{C})\right)$ and $\mathcal{O}_{q}\left(M_{n}(\mathbb{C})\right)$.

\section{CASE OF $\mathcal{O}_{q}\left(S L_{2}(\mathbb{C})\right)$}

In this section, we prove Theorem 1.2 for $\mathcal{O}_{q}\left(S L_{2}(\mathbb{C})\right)$ which is the base step of the induction that we use in the proof of the general case. In fact, in the induction step we will show the statement for $\mathcal{O}_{q}\left(M_{n}(\mathbb{C})\right)$ and not for $\mathcal{O}_{q}\left(S L_{n}(\mathbb{C})\right)$ but in the light of Proposition 3.1 these are equivalent. The only reason why we use $S L_{2}$ in this part and not $M_{2}$ is that $\mathcal{O}_{q}\left(S L_{2}(\mathbb{C})\right.$ ) has fewer elements (in the sense of Gelfand-Kirillov dimension) so the computations are shorter.

Proposition 4.1. Assume that $q \in \mathbb{C}^{\times}$is not a root of unity. The centralizer of $\bar{\sigma}_{1} \in \mathcal{O}_{q}\left(S L_{2}(\mathbb{C})\right)$ is $\mathbb{C}\left\langle\sigma_{1}\right\rangle$.

For simplicity, we will use the notations $a:=x_{1,1}+\left(\operatorname{det}_{q}-1\right), b:=x_{1,2}+\left(\operatorname{det}_{q}-1\right)$, $c:=x_{2,1}+\left(\operatorname{det}_{q}-1\right)$ and $d:=x_{2,2}+\left(\operatorname{det}_{q}-1\right)$ for the generators of $\mathcal{O}_{q}\left(S L_{2}(\mathbb{C})\right)$. In particular, $\bar{\sigma}_{1}=a+d$. 
By Theorem I.7.16. in $\left[\mathrm{BG}\right.$ we have a basis of $\mathcal{O}_{q}\left(S L_{2}(\mathbb{C})\right)$ consisting of the following elements:

$$
a^{i} b^{k} c^{l}, b^{k} c^{l} d^{j}, b^{k} c^{l} \quad\left(i, j \in \mathbb{N}^{+}, k, l \in \mathbb{N}\right)
$$

We will use the $\mathbb{Z} / 2 \mathbb{Z}$-grading of $\mathcal{O}_{q}\left(S L_{2}(\mathbb{C})\right)$ defined as $\operatorname{deg}\left(a^{i} b^{k} c^{l}\right)=i \bmod 2$ and $\operatorname{deg}\left(b^{k} c^{l} d^{j}\right)=j \bmod 2$. Note, that it is not the $\mathbb{Z} / 2 \mathbb{Z}$-grading that it inherits from the $\mathbb{Z}$-grading of $\mathcal{O}_{q}\left(M_{2}(\mathbb{C})\right)$ which would be $i+k+l$ and $k+l+j$ modulo 2. Still, this is a grading in the sense of graded algebras.

Proof. First, let us compute the action of $\bar{\sigma}_{1}=a+d$ on the basis elements:

$$
\begin{aligned}
(a+d) \cdot a^{i} b^{k} c^{l} & =a^{i+1} b^{k} c^{l}+\left(1+q^{-1} b c\right) a^{i-1} b^{k} c^{l}= \\
& =a^{i+1}\left(b^{k} c^{l}\right)+a^{i-1}\left(b^{k} c^{l}+q^{-2(i-1)-1} b^{k+1} c^{l+1}\right)
\end{aligned}
$$

and similarly,

$$
\begin{aligned}
a^{i} b^{k} c^{l} \cdot(a+d) & =q^{-(k+l)} a^{i+1} b^{k} c^{l}+q^{k+l} a^{i-1}(1+q b c) b^{k} c^{l}= \\
& =a^{i+1}\left(q^{-(k+l)} b^{k} c^{l}\right)+a^{i-1}\left(q^{k+l} b^{k} c^{l}+q^{k+l+1} b^{k+1} c^{l+1}\right)
\end{aligned}
$$

Hence, for the commutator, we get

$$
\begin{aligned}
{\left[(a+d), a^{i} b^{k} c^{l}\right] } & =a^{i+1}\left(\left(1-q^{-(k+l)}\right) b^{k} c^{l}\right)+ \\
& +a^{i-1}\left(\left(1-q^{k+l}\right) b^{k} c^{l}+\right. \\
& \left.+\left(q^{-2(i-1)-1}-q^{k+l+1}\right) b^{k+1} c^{l+1}\right)
\end{aligned}
$$

By the same computation on $b^{k} c^{l} d^{j}$ and $b^{k} c^{l}$, one can conclude that

$$
\begin{aligned}
{\left[(a+d), b^{k} c^{l} d^{j}\right] } & =\left(\left(q^{-(k+l)}-1\right) b^{k} c^{l}\right) d^{j+1}+ \\
& +\left(\left(q^{k+l}-1\right) b^{k} c^{l}+\right. \\
& \left.+\left(q^{k+l+1}-q^{-2(j-1)-1}\right) b^{k+1} c^{l+1}\right) d^{j-1} \\
{\left[(a+d), b^{k} c^{l}\right]=} & a\left(1-q^{-(k+l)}\right) b^{k} c^{l}+\left(q^{-(k+l)}-1\right) b^{k} c^{l} d
\end{aligned}
$$

Generally, for a polynomial $p \in \mathbb{C}\left[t_{1}, t_{2}\right]$ and $i \geq 1$ :

$$
\begin{aligned}
{\left[(a+d), a^{i} p(b, c)\right] } & =a^{i+1} \sum_{m}\left(\left(1-q^{-m}\right) p_{m}(b, c)\right)+ \\
& +a^{i-1}\left(\sum_{m}\left(1-q^{m}\right) p_{m}(b, c)+\right. \\
& \left.+\left(q^{-2(i-1)-1}-q^{m+1}\right) b c \cdot p_{m}(b, c)\right)
\end{aligned}
$$

where $p_{m}$ is the $m$-th homogeneous component of $p$ with respect to the $\mathbb{N}$-valued total degree on $\mathbb{C}\left[t_{1}, t_{2}\right] \cong \mathbb{C}\langle b, c\rangle$. The analogous computations for $p(b, c) d^{j}(j \geq 1)$ and $p(b, c)$ give

$$
\begin{aligned}
{\left[(a+d), p(b, c) d^{j}\right] } & =\sum_{m}\left(\left(q^{-m}-1\right) p_{m}(b, c)\right) d^{j+1}+ \\
& +\sum_{m}\left(\left(q^{m}-1\right) p_{m}(b, c)+\right. \\
& \left.+\left(q^{m+1}-q^{-2(j-1)-1}\right) b c p_{m}(b, c)\right) d^{j-1}
\end{aligned}
$$




$$
[(a+d), p(b, c)]=a \sum_{m}\left(\left(1-q^{-m}\right) p_{m}(b, c)\right)+\sum_{m}\left(\left(q^{-m}-1\right) p_{m}(b, c)\right) d
$$

To prove the statement, it is enough to show that in each subspace $\sum_{i=0}^{\alpha} a^{i}$. $\mathbb{C}\langle b, c\rangle+\sum_{j=0}^{\alpha} \mathbb{C}\langle b, c\rangle \cdot d^{i} \leq \mathcal{O}_{q}\left(S L_{2}(\mathbb{C})\right)$ the space of $\bar{\sigma}_{1}$-centralizing elements has dimension $\alpha+1$. Indeed, $\sum_{i=0}^{\alpha} \mathbb{C} \bar{\sigma}_{1}^{i}$ has exactly dimension $\alpha+1$ by $\mathbb{C}\left\langle\bar{\sigma}_{1}\right\rangle \cong \mathbb{C}[t]$, and these are $\bar{\sigma}_{1}$-centralizing elements, so then there cannot be anything else that commutes with $\bar{\sigma}_{1}$.

Assume that the nonzero element $g$ commutes with $\bar{\sigma}_{1}$ and express $g$ in the above mentioned basis as:

$$
g=\sum_{i=1}^{\alpha} a^{i} r_{i}+\sum_{j=1}^{\beta} s_{j} d^{j}+u
$$

where $r_{i}, s_{j}$ and $u$ are elements of $\mathbb{C}\langle b, c\rangle$, and $\alpha$ and $\beta$ are the highest powers of $a$ and $d$ appearing in the decomposition (i.e. $r_{\alpha} \neq 0$ and $s_{\beta} \neq 0$ ). We will also write $r_{0}$ or $s_{0}$ for $u$, if it makes the formula simpler. Since $\bar{\sigma}_{1}$ is a homogeneous element with respect to the $\mathbb{Z} / 2 \mathbb{Z}$-grading, we may assume that $g$ is also homogeneous.

The proof is split into two cases: if $g$ has degree $0 \in \mathbb{Z} / 2 \mathbb{Z}$ (hence $\alpha$ is even) then we will prove that the constant terms of the $\frac{\alpha}{2}+1$ polynomials $r_{\alpha}, r_{\alpha-2}, \ldots, r_{2}, u \in$ $\mathbb{C}[b, c]$ determine $g$ uniquely, and similarly, if $g \in \mathbb{Z} / 2 \mathbb{Z}$ has degree 1 (hence $\alpha$ is odd) then the constant terms of the $\frac{\alpha+1}{2}$ polynomials $r_{\alpha}, r_{\alpha-2}, \ldots, r_{1} \in \mathbb{C}[b, c]$ also determine $g$ uniquely. This is enough, since then in the even case, we get $\left(\frac{\alpha}{2}+1\right)+\frac{(\alpha-1)+1}{2}=\alpha+1$ for the dimension of the $\bar{\sigma}_{1}$-centralizing elements as the sum of dimensions of homogeneous $\bar{\sigma}_{1}$-centralizing elements in even and odd degrees. Similarly, if $\alpha$ is odd, it is $\frac{\alpha+1}{2}+\frac{\alpha-1}{2}=\alpha+1$ so it is indeed enough to prove the above claim.

First, we prove that $r_{\alpha} \in \mathbb{C} \cdot 1$ in both cases. If $\alpha=0$ then $r_{\alpha}=u$ so the $a^{i} b^{k} c^{l}$ terms in $[a+d, g]$ (decomposed in the monomial basis) are the same as the $a^{i} b^{k} c^{l}$ terms in $[a+d, u]$ by 4.2 , 4.3 and 4.4. However, by 4.4, these terms would be nonzero if $u \notin \mathbb{C}$. Now, assume that $\alpha \geq 1$ and define the subspace

$$
\mathcal{A}^{d}:=\operatorname{Span}_{\mathbb{C}}\left(a^{i} b^{k} c^{l}, b^{k} c^{l} d^{j}, b^{k} c^{l} \mid i \leq d, k, l \in \mathbb{N}\right)
$$

for any $d \in \mathbb{N}$. Then, by the fact that $\bar{\sigma}_{1} \mathcal{A}^{\alpha-1}, \mathcal{A}^{\alpha-1} \bar{\sigma}_{1}, d \mathcal{A}^{\alpha}$ and $\mathcal{A}^{\alpha} d$ are all contained in $\mathcal{A}^{\alpha}$ (using the defining relations), we have

$\bar{\sigma}_{1} g-g \bar{\sigma}_{1}+\mathcal{A}^{\alpha} \subseteq \bar{\sigma}_{1}\left(a^{\alpha} r_{\alpha}+\mathcal{A}^{\alpha-1}\right)-\left(a^{\alpha} r_{\alpha}+\mathcal{A}^{\alpha-1}\right) \bar{\sigma}_{1}+\mathcal{A}^{\alpha}=a \cdot a^{\alpha} r_{\alpha}-a^{\alpha} r_{\alpha} \cdot a+\mathcal{A}^{\alpha}$

Moreover, if $r_{\alpha}=\sum \lambda_{k, l} b^{k} c^{l}$ then $a^{\alpha} r_{\alpha} \cdot a=\sum \lambda_{k, l} q^{-k-l} a^{\alpha+1} b^{k} c^{l}$. Since the elements $a^{\alpha+1} b^{k} c^{l}$ are independent even modulo $\mathcal{A}^{\alpha}$ by Section $2.3, a^{\alpha} r_{\alpha} \cdot a$ can agree with $a^{\alpha+1} r_{\alpha}$ modulo $\mathcal{A}^{\alpha}$ only if $\lambda_{k, l}=0$ for all $(k, l) \neq(0,0)$. Therefore, $r_{\alpha} \in \mathbb{C} \cdot 1$.

Now, we prove that for all $1 \leq i \leq \alpha-1, r_{i+1}$ and the constant term of $r_{i-1}$ determines $r_{i-1} \in \mathbb{C}[b, c]$. Indeed, by equation 4.2 we have

$$
\begin{aligned}
0=\operatorname{Coeff}_{a^{i}}([(a+d), g]) & =\sum_{m}\left(\left(1-q^{-m}\right) r_{i-1, m}\right)+ \\
& +\sum_{m}\left(1-q^{m}\right) r_{i+1, m}+ \\
& +\sum_{\substack{m \\
8}}\left(q^{-2(i-1)-1}-q^{m+1}\right) b c \cdot r_{i+1, m}
\end{aligned}
$$


where and $r_{i, m}$ is the $m$-th homogeneous term of $r_{i} \in \mathbb{C}[b, c]$ and Coeff $a_{a^{i}}$ stands for the element in $\mathbb{C}[b, c]$ such that $a^{i} \cdot \operatorname{Coeff}_{a^{i}}(x)$ is a summand of $x$ when it is decomposed in the monomial basis. The degree $k$ part of the right hand side is

$$
\begin{gathered}
\left(1-q^{-k}\right) r_{i-1, k}+\left(1-q^{k}\right) r_{i+1, k}+\left(q^{1-2 i}-q^{k-1}\right) b c \cdot r_{i+1, k-2} \quad \text { if } k \geq 2 \\
\left(1-q^{-1}\right) r_{i-1,1}+\left(1-q^{1}\right) r_{i+1,1} \quad \text { if } k=1
\end{gathered}
$$

for all $1 \leq i \leq \alpha-1$. Hence $r_{i+1}$ determines $r_{i-1}$ (using that $q$ is not a root of unity) except for the constant term $r_{i-1,0}$ which has zero coefficient in 4.5 for all $k$.

We prove that $\operatorname{deg} s_{j+1} \leq \operatorname{deg} s_{j-1}-2$ for all $j \geq 1$ where deg stands for the total degree of $\mathbb{C}[b, c]$. Analogously to 4.5 , one can deduce the following by 4.3 .

$$
\begin{aligned}
0=\operatorname{Coeff}_{d^{j}}([(a+d), g]) & =\sum_{m}\left(\left(q^{-m}-1\right) s_{j-1, m}\right)+ \\
& +\sum_{m}\left(q^{m}-1\right) s_{j+1, m} \\
& +\sum_{m}\left(q^{m+1}-q^{-2(j-1)-1}\right) b c \cdot s_{j+1, m}
\end{aligned}
$$

The degree $k$ part of the right hand side is

$$
\begin{gathered}
\left(q^{-k}-1\right) s_{j-1, k}+\left(q^{k}-1\right) s_{j+1, k}+\left(q^{k-1}-q^{1-2 j}\right) b c \cdot s_{j+1, k-2} \quad \text { if } k \geq 2 \\
\left(q^{-1}-1\right) s_{j-1,1}+\left(q^{1}-1\right) s_{j+1,1} \quad \text { if } k=1
\end{gathered}
$$

for all $1 \leq j \leq \beta-1$. Note that $q^{k-1}-q^{1-2 j}=0$ can never happen for $k \geq 2$. If $s_{j+1}=0$ then the statement is empty. If $s_{j+1} \neq 0$ then for $k=2+\operatorname{deg} s_{j+1} \geq 2$, we have $s_{j+1, k}=0$ but $s_{j+1, k-2}=s_{j+1, \operatorname{deg} s_{j+1}} \neq 0$ hence 4.6 gives $s_{j-1, k} \neq 0$. So $\operatorname{deg} s_{j+1} \leq \operatorname{deg} s_{j-1}-2$.

Now, assume that $\alpha$ is even. By the previous paragraphs, the scalars $r_{\alpha}$, $r_{\alpha-2,0}, \ldots, r_{2,0}$ and $u_{0}$ determine all the polynomials $r_{\alpha}, r_{\alpha-2}, r_{\alpha-4}, \ldots, r_{2}$ and $u$. We prove that they also determine the $s_{j}$ 's. Starting from $u=s_{0}$ one can obtain $s_{j+1}$ by $s_{j-1}$. Indeed, since $\operatorname{deg} s_{j+1} \leq \operatorname{deg} s_{j-1}-2$, if $\operatorname{deg} s_{j-1} \leq 1$ then $s_{j+1}=0$, and similarly, for $k=\operatorname{deg} s_{j-1} \geq 2$ we have $s_{j+1, k-1}=0$ and 4.6 gives $\left(q^{-k}-1\right) s_{j-1, k}=-\left(q^{k-1}-q^{1-2 j}\right) b c \cdot s_{j+1, k-2}$. Then, recursively for $k$, if $s_{j-1, k}$ and $s_{j+1, k}$ are given, by 4.6 they determine $s_{j+1, k-2}$, using that $q$ is not a root of unity.

If $\alpha$ is odd, then by 4.2 one can obtain the following for the summand of $[(a+d), g]$ that does not contain $a$ and $d$ when decomposed in the given basis:

$$
\begin{aligned}
0=\operatorname{Coeff}_{1}([(a+d), g]) & =\sum_{m}\left(\left(1-q^{m}\right) r_{1, m}+\left(q-q^{m+1}\right) b c \cdot r_{1, m}+\right. \\
& \left.+\left(q^{m}-1\right) s_{1, m}+\left(q^{m+1}-q\right) b c \cdot s_{1, m}\right)
\end{aligned}
$$

The homogeneous components of degree $k$ are

$$
\begin{aligned}
& \left(1-q^{k}\right) r_{1, k}+\left(q-q^{k-1}\right) b c \cdot r_{i+1, k-2}+\left(q^{k}-1\right) s_{1, k}+\left(q^{k+1}-q\right) b c \cdot s_{1, k-2} \quad \text { if } k \geq 2 \\
& (1-q) r_{1,1}+(q-1) s_{1,1} \quad \text { if } k=1
\end{aligned}
$$


Hence, $r_{\alpha}, r_{\alpha-2,0}, \ldots, r_{1,0}$ determine not only $r_{i}$ for $1 \leq i \leq \alpha$ but also $s_{1}$ by 4.7 applied for $k=\operatorname{deg} s_{1}+2$ and the same recursive argument as in the even case. Then, similarly, $s_{j+1}$ is unique by $s_{j-1}$ for all $2 \leq j \leq \beta-1$ and the statement follows.

\section{Proof of the MAIN RESUlt}

In [DL1, to verify that the subalgebra of cocommutative elements in $A_{n}:=$ $\mathcal{O}_{q}\left(M_{n}(\mathbb{C})\right)$ is generated by the $\sigma_{i}$ 's, they proved that the natural surjection

$$
\eta: \mathcal{O}_{q}\left(M_{n}(\mathbb{C})\right) \rightarrow \mathbb{C}\left[t_{1}, \ldots, t_{n}\right] \quad x_{i, j} \mapsto \delta_{i, j} t_{i}
$$

restricted to the subalgebra of cocommutative elements $\mathcal{O}_{q}\left(M_{n}(\mathbb{C})\right)^{\text {coc }}$ is an isomorphism and its image is the subalgebra of symmetric polynomials $D_{n}^{S_{n}}$ where $D_{n}:=\mathbb{C}\left[t_{1}, \ldots, t_{n}\right]$. We use the same plan to prove that it is also the centralizer of $\sigma_{1} \in \mathcal{O}_{q}\left(M_{n}(\mathbb{C})\right)$.

For this purpose, we will need the following intermediate quotient algebra between $A_{n}$ and $D_{n}$ :

$$
B_{2, n}:=A_{n} /\left(x_{1, j}, x_{i, 1} \mid 2 \leq i, j \leq n\right)
$$

Let us denote the corresponding natural surjection by $\varphi: A_{n} \rightarrow B_{2, n}$. Since $\operatorname{Ker} \eta \subseteq \operatorname{Ker} \varphi$ by their definition, $\eta$ can be factored through $\varphi$. So our setup is:

$$
C\left(\sigma_{1}\right) \subseteq A_{n} \stackrel{\varphi}{\longrightarrow} B_{2, n} \stackrel{\delta}{\longrightarrow} D_{n}
$$

where $\eta=\delta \circ \varphi$ and $C\left(\sigma_{1}\right)$ denotes the centralizer of $\sigma_{1}$ in $A_{n}$. The structure of $B_{2, n}$ is quite simple: $B_{2, n} \cong A_{n-1}[t]$ by the map $x_{i, j} \mapsto x_{i-1, j-1}$ for $i, j \geq 2$ and $x_{1,1} \mapsto t$. One can check that it is indeed an isomorphism since $x_{1,1}$ commutes with the elements of $\mathbb{C}\left\langle x_{1,1}, x_{i, j} \mid i, j \geq 2\right\rangle$ modulo $\operatorname{Ker} \varphi$.

These algebras are $\mathbb{N}$-graded algebras using the total degree of $A_{n}$, but we can also endow them by a filtration that is not the corresponding filtration of the grading. Namely, for each $d \in \mathbb{N}$ let $\mathcal{A}^{d}$ be the subspace of $A_{n}$ that is generated by the monomials in which $x_{1,1}$ appears at most $d$ times, i.e. it is spanned by the ordered monomials of the form $x_{1,1}^{i} m$ where $i \leq d$ and $m$ is an ordered monomial in the variables $x_{i, j},(i, j) \neq(1,1)$. One can check that this is indeed a filtration: they are linear subspaces such that $\cup_{d} \mathcal{A}^{d}=A_{n}$ and $\mathcal{A}^{d} \cdot \mathcal{A}^{e} \subseteq \mathcal{A}^{d \cdot e}$ for all $d, e \in \mathbb{N}$. As $C\left(\sigma_{1}\right)$ is a subalgebra of $A_{n}$, we get an induced filtration $\mathcal{C}^{d}=\mathcal{A}^{d} \cap C\left(\sigma_{1}\right)(d \in \mathbb{N})$ on $C\left(\sigma_{1}\right)$, and similarly, an induced filtration $\mathcal{B}^{d}:=\varphi\left(\mathcal{A}^{d}\right)(d \in \mathbb{N})$ on $B_{2, n}$ and $\mathcal{D}^{d}:=\delta \circ \varphi\left(\mathcal{A}^{d}\right)(d \in \mathbb{N})$ on $D_{n}$.

Proof of Theorem 1.2. We prove the statement by induction on $n$. The statement is verified for $\mathcal{O}_{q}\left(S L_{2}(\mathbb{C})\right)$ in Section 4 so by Proposition 3.1 the case $n=2$ is proved. Now, assume that $n \geq 3$. We shall prove that

- $\left.(\delta \circ \varphi)\right|_{C\left(\sigma_{1}\right)}: C\left(\sigma_{1}\right) \rightarrow D_{n}$ is injective, and

- the image $(\delta \circ \varphi)\left(C\left(\sigma_{1}\right)\right)$ is in $D_{n}^{S_{n}}$.

This means that the restriction of $\delta \circ \varphi$ to $C\left(\sigma_{1}\right)$ yields an isomorphism with $D_{n}^{S_{n}}$, since by DL1, $C\left(\sigma_{1}\right) \ni \sigma_{i}$ for all $i=1, \ldots, n$ and $\delta \circ \varphi$ restricts to an isomorphism between $\mathbb{C}\left\langle\sigma_{1}, \ldots, \sigma_{n}\right\rangle$ and $D_{n}^{S_{n}}$.

First part, step 1: First, we show that it is enough to prove that $\operatorname{gr}(\delta \circ \varphi)$ restricted to $\operatorname{gr}\left(C\left(\sigma_{1}\right)\right)$ is injective to get the injectivity of $\delta \circ \varphi$ on $C\left(\sigma_{1}\right)$. Apply 
gr to the filtered algebras in our setup presented in Diagram 5.1. It gives

$$
\operatorname{gr}\left(C\left(\sigma_{1}\right)\right) \subseteq \operatorname{gr}\left(A_{n}\right) \stackrel{\operatorname{gr}(\varphi)}{\longrightarrow} \operatorname{gr}\left(B_{2, n}\right) \stackrel{\operatorname{gr}(\delta)}{\longrightarrow} \operatorname{gr}\left(D_{n}\right)
$$

The surjectivity of the maps follow by $\varphi\left(\mathcal{A}^{d}\right)=\mathcal{B}^{d}$ and $\delta\left(\mathcal{B}^{d}\right)=\mathcal{D}^{d}$. Assuming that $\operatorname{gr}(\delta \circ \varphi)$ restricted to $\operatorname{gr}\left(C\left(\sigma_{1}\right)\right)$ is injective, we get the injectivity of $\left.(\delta \circ \varphi)\right|_{\mathcal{C}^{0}}$, moreover, we can apply an induction on $d$ using the 5-lemma in the following commutative diagram of vector spaces for all $d \geq 1$ :

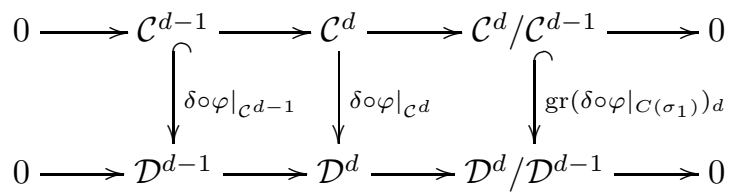

where the rows are exact by definition and $\operatorname{gr}\left(\left.\delta \circ \varphi\right|_{C\left(\sigma_{1}\right)}\right)_{d}$ and $\left.\delta \circ \varphi\right|_{\mathcal{C}^{d-1}}$ are injective by the assumption and the induction hypothesis. Therefore, $\delta \circ \varphi$ is injective on $\cup_{d} \mathcal{C}^{d}=C\left(\sigma_{1}\right)$.

Notice that $B_{2, n}$ and $D_{n}$ are not only filtered by the $\varphi\left(x_{1,1}\right)$ and $t_{1}$ degrees but they are also graded as $B_{2, n} \cong A_{n-1}[t]$ and $D_{n} \cong D_{n-1}[t]$ by $t_{1} \mapsto t$ and $t_{i} \mapsto t_{i-1} \in D_{n-1}(i \geq 2)$. Hence, we will use the natural identifications of graded algebras $B_{2, n} \cong \operatorname{gr}\left(B_{2, n}\right)$ and $\operatorname{gr}\left(D_{n}\right) \cong D_{n}$ (and so $\operatorname{gr}(\delta)$ is just $\left.\delta\right)$.

Step 2: We prove that the image of the map $\operatorname{gr}(\varphi)$ restricted to $\operatorname{gr}\left(C\left(\sigma_{1}\right)\right)$ is in $C\left(\varphi\left(\sigma_{1}\right)\right) \subseteq B_{2, n}$. Here, $C\left(\varphi\left(\sigma_{1}\right)\right)$ is a graded subalgebra of $B_{2, n}$ since $\varphi\left(\sigma_{1}\right)$ is a sum of a central element $\varphi\left(x_{1,1}\right)$ and of the elements $\varphi\left(x_{2,2}\right), \ldots, \varphi\left(x_{n, n}\right)$ (that are homogeneous of degree zero) so $C\left(\varphi\left(\sigma_{1}\right)\right)=C\left(\varphi\left(x_{2,2}+\cdots+x_{n, n}\right)\right)$ is homogeneous. The proof of this step is clear: For an $h \in \mathcal{C}^{d} \subseteq \mathcal{A}^{d}$ we have $0=\varphi\left(\left[\sigma_{1}, h\right]\right)=$ $\left[\varphi\left(\sigma_{1}\right), \varphi(h)\right]$, hence $\operatorname{gr}(\varphi)\left(h+\mathcal{C}^{d-1}\right) \in C\left(\varphi\left(\sigma_{1}\right)\right)$.

Step 3: We prove the injectivity of $\operatorname{gr}(\delta)$ restricted to $C\left(\varphi\left(\sigma_{1}\right)\right)$ by the induction. First, note that $C\left(\varphi\left(\sigma_{1}\right)\right) \cong C_{A_{n-1}}\left(\sigma_{1}\right)[t]$ using the isomorphism $B_{2, n} \cong A_{n-1}[t]$. Then, by the induction hypothesis,

$$
C_{A_{n-1}}\left(\sigma_{1}\right)=\mathbb{C}\left\langle\sigma_{1}\left(A_{n-1}\right), \ldots, \sigma_{n-1}\left(A_{n-1}\right)\right\rangle
$$

Therefore, $C\left(\varphi\left(\sigma_{1}\right)\right)=\mathbb{C}\left\langle\sigma_{1}\left(B_{2, n}\right), \ldots, \sigma_{n-1}\left(B_{2, n}\right), \varphi\left(x_{1,1}\right)\right\rangle$ where $\sigma_{i}\left(B_{2, n}\right)$ is defined as the image of $\sigma_{i}\left(A_{n-1}\right)$ under the above mentioned isomorphism. For these elements, we have $\delta\left(\sigma_{i}\left(B_{2, n}\right)\right)=s_{i}\left(t_{2}, \ldots, t_{n}\right)$ where $s_{i}\left(t_{2}, \ldots, t_{n}\right)$ is the $i$-th elementary symmetric polynomial in the variables $t_{2}, \ldots, t_{n}$. Hence, $\delta$ is indeed injective by the fundamental theorem of symmetric polynomials. Now, it is enough to prove the injectivity of $\operatorname{gr}(\varphi)$ restricted to $C\left(\sigma_{1}\right)$ to get the injectivity of $\delta \circ \varphi$ by Step 1 and 2 .

Step 4: For ad $\sigma_{1}: A_{n} \rightarrow A_{n}, h \mapsto\left[\sigma_{1}, h\right]$, we have $C\left(\sigma_{1}\right)=\operatorname{Ker}\left(\operatorname{ad} \sigma_{1}\right)$ by definition. Although ad $\sigma_{1}$ is not a morphism of algebras but a derivation of degree 1 , we can still take

$$
\operatorname{Ker}\left(\operatorname{gr}\left(\operatorname{ad} \sigma_{1}\right)\right):=\left\{\left(h_{d}\right)_{d \in \mathbb{N}} \in \operatorname{gr}\left(A_{n}\right) \mid \sigma_{1} h_{d}-h_{d} \sigma_{1}+\mathcal{A}^{d}=0 \in \mathcal{A}^{d+1} / \mathcal{A}^{d}\right\}
$$


where $\operatorname{gr}\left(\operatorname{ad} \sigma_{1}\right)$ is understood as a map of graded vector spaces. Then, we can extend Diagram 5.2 as:

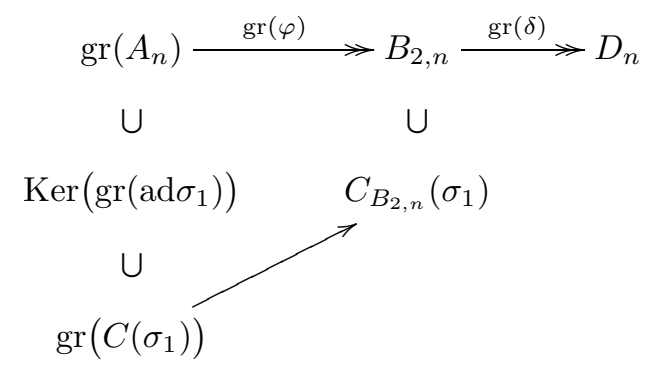

Naturally, $\operatorname{gr}\left(C\left(\sigma_{1}\right)\right) \subseteq \operatorname{Ker}\left(\operatorname{gr}\left(\operatorname{ad} \sigma_{1}\right)\right)$ since $\sigma_{1} h_{d}-h_{d} \sigma_{1}=0 \in A_{n}$ implies $\sigma_{1} h_{d}-$ $h_{d} \sigma_{1} \in \mathcal{A}^{d}$.

We give an explicit description of $\operatorname{Ker}\left(\operatorname{gr}\left(\operatorname{ad} \sigma_{1}\right)\right)$. Observe that

$$
\operatorname{gr}\left(A_{n}\right) \cong \bigoplus_{d \in \mathbb{N}} y^{d} \mathbb{C}\left\langle x_{i, j} \mid(i, j) \neq(1,1)\right\rangle
$$

where $y$, the image of $x_{1,1}$, commutes with every $x_{i, j}$ for $2 \leq i, j \leq n$ and $q$ commutes with $x_{1, j}$ and $x_{i, 1}$ for all $i, j \geq 2$. Indeed, by the monomial basis of $A_{n}$ (see Section 2) we get the direct sum decomposition, moreover, the only defining relations involving $x_{1,1}$ are $x_{1,1} x_{1, j}=q x_{1, j} x_{1,1}, x_{1,1} x_{i, 1}=q x_{i, 1} x_{1,1}$ and $x_{1,1} x_{i, j}=$ $x_{i, j} x_{1,1}+\left(q-q^{-1}\right) x_{i, 1} x_{1, j}$ that reduce to $q$-commutativity of $y$ and commutativity of $y$ with the appropriate elements. The argument also gives that the image of the monomial basis of $A_{n}$ is a monomial basis in $\operatorname{gr}\left(A_{n}\right)$.

In particular, we get that

$$
\operatorname{Ker}\left(\operatorname{gr}\left(\operatorname{ad} \sigma_{1}\right)\right) \cong \bigoplus_{d \in \mathbb{N}} y^{d} \mathbb{C}\left\langle x_{i, j} \mid 2 \leq i, j \leq n\right\rangle
$$

by the same isomorphism. Indeed, for an element $x_{1,1}^{d} m \in \mathcal{A}^{d}$ where $m$ is an ordered monomial in the variables $x_{i, j}((i, j) \neq(1,1))$, we have

$$
\operatorname{gr}\left(\operatorname{ad} \sigma_{1}\right)\left(x_{1,1}^{d} m+\mathcal{A}^{d-1}\right)=x_{1,1} \cdot x_{1,1}^{d} m-x_{1,1}^{d} m \cdot x_{1,1}+\mathcal{A}^{d}
$$

since $x_{i, i} \cdot \mathcal{A}^{d} \subseteq \mathcal{A}^{d}$ and $\mathcal{A}^{d} \cdot x_{i, i} \subseteq \mathcal{A}^{d}$ for all $i \geq 2$. Then, by the above mentioned $q$-commutativity relations, we get $\left(1-q^{-c(m)}\right) x_{1,1}^{d+1} m+\mathcal{A}^{d}$ where $c(m)$ stands for the sum of exponents of the $x_{1, j}$ 's and $x_{i, 1}$ 's $(2 \leq i, j \leq n)$ appearing in $m$. The result is a monomial basis element in $\mathcal{A}^{d+1} / \mathcal{A}^{d} \subseteq \operatorname{gr}\left(A_{n}\right)$. For different monomials $x_{1,1}^{d} m$ and $x_{1,1}^{d^{\prime}} m^{\prime}$ we get different monomials $x_{1,1}^{d+1} m$ and $x_{1,1}^{d^{\prime}+1} m^{\prime}$ so $\operatorname{gr}\left(\operatorname{ad} \sigma_{1}\right)$ is diagonal in the monomial basis of $\operatorname{gr}\left(A_{n}\right)$ with the scalars $\left(1-q^{-c(m)}\right)$. Hence, its kernel is $\left\{x_{11}^{d} m+\mathcal{A}^{d-1} \mid d \in \mathbb{N}, c(m)=0\right\}$ since $q$ is not a root of unity, as we stated. Therefore, we get that $\operatorname{Ker}\left(\operatorname{gr}\left(\operatorname{ad} \sigma_{1}\right)\right) \cong A_{n-1}[t]$ using $y \mapsto t$ and $x_{i, j} \mapsto x_{i-1, j-1}$ since $y$ commutes with every $x_{i, j}$ for $2 \leq i, j \leq n$.

Now, the injectivity part of the theorem follows: the isomorphisms $B_{2, n} \cong$ $A_{n-1}[t]$ and $\operatorname{Ker}\left(\operatorname{gr}\left(\operatorname{ad} \sigma_{1}\right)\right) \cong A_{n-1}[t]$ established in step 4 are compatible, meaning that $\operatorname{gr}(\varphi)$ composed with them on the appropriate sides is $\operatorname{id}_{A_{n-1}[t]}$. In particular, $\operatorname{gr}(\varphi)$ restricted to $\operatorname{gr}\left(C\left(\sigma_{1}\right)\right) \subseteq \operatorname{Ker}\left(\operatorname{gr}\left(\operatorname{ad} \sigma_{1}\right)\right)$ is injective. By step $3, \operatorname{gr}(\delta)$ restricted to $C\left(\varphi\left(\sigma_{1}\right)\right)$ is also injective, so the composition $\delta \circ \operatorname{gr}(\varphi)=\operatorname{gr}(\delta \circ \varphi)$ is injective as well, using step 2. By step 1 , this means that $\delta \circ \varphi$ is injective. 
Second part: To prove $\eta\left(C\left(\sigma_{1}\right)\right) \subseteq D_{n}^{S_{n}}$, consider the following commutative diagram:

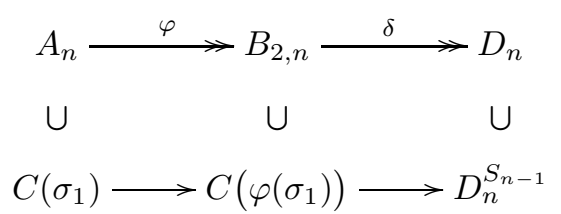

where $S_{n-1}$ acts on $D_{n}$ by permuting $t_{2}, \ldots, t_{n}$. The diagram implicitly states that $\varphi\left(C\left(\sigma_{1}\right)\right) \subseteq C\left(\varphi\left(\sigma_{1}\right)\right)$ (which is clear) and that $\delta\left(C\left(\varphi\left(\sigma_{1}\right)\right)\right) \subseteq D_{n}^{S_{n-1}}$. The latter follows by the induction hypothesis for $n-1$ : it gives that $C\left(\varphi\left(\sigma_{1}\right)\right)=$ $\mathbb{C}\left\langle\sigma_{1}\left(B_{2, n}\right), \ldots, \sigma_{n-1}\left(B_{2, n}\right), \varphi\left(x_{1,1}\right)\right\rangle$ by $B_{2, n} \cong A_{n-1}[t]$ and since $\delta\left(\varphi\left(x_{1,1}\right)\right)=t_{1}$ and $\delta\left(\sigma_{i}\left(B_{2, n}\right)\right)=s_{i}\left(t_{2}, \ldots, t_{n}\right)$, the $i$-th elementary symmetric polynomial in the variables $t_{2}, \ldots, t_{n}$, we get that $(\delta \circ \varphi)\left(C\left(\sigma_{1}\right)\right)$ is symmetric in $t_{2}, \ldots, t_{n}$.

To prove symmetry in $t_{1}, \ldots, t_{n-1}$ too, consider the isomorphism $\gamma: \mathcal{O}_{q}\left(M_{n}(\mathbb{C})\right) \cong$ $\mathcal{O}_{q^{-1}}\left(M_{n}(\mathbb{C})\right)$ given by $x_{i, j} \leftrightarrow x_{n+1-i, n+1-j}^{\prime}$ where $x_{i, j}^{\prime}$ denotes the variables in $\mathcal{O}_{q^{-1}}\left(M_{n}(\mathbb{C})\right)$. This is indeed an isomorphism: interpreted in the free algebra it maps the defining relations of $\mathcal{O}_{q}\left(M_{n}(\mathbb{C})\right)$ to the defining relations of $\mathcal{O}_{q^{-1}}\left(M_{n}(\mathbb{C})\right)$. It also maps $\sigma_{1} \in \mathcal{O}_{q}\left(M_{n}(\mathbb{C})\right)$ into the $\sigma_{1}$ of $\mathcal{O}_{q^{-1}}\left(M_{n}(\mathbb{C})\right)$ denoted by $\sigma_{1}^{\prime}$. Moreover, $\bar{\gamma} \circ \eta=\eta^{\prime} \circ \gamma$ where $\bar{\gamma}: D_{n} \rightarrow D_{n}, t_{i} \mapsto t_{n+1-i}(i=1, \ldots, n)$ and $\eta^{\prime}: \mathcal{O}_{q^{-1}}\left(M_{n}(\mathbb{C})\right) \rightarrow \mathbb{C}\left[t_{1}, \ldots, t_{n}\right], x_{i, j}^{\prime} \mapsto t_{i} \delta_{i, j}$ is the $\eta(=\delta \circ \varphi)$ of $\mathcal{O}_{q^{-1}}\left(M_{n}(\mathbb{C})\right)$. Hence, $(\bar{\gamma} \circ \eta)\left(C\left(\sigma_{1}\right)\right)=\eta^{\prime}\left(C\left(\sigma_{1}^{\prime}\right)\right)$ as $C\left(\sigma_{1}^{\prime}\right)$ is symmetric under $\bar{\gamma}$. Applying the previous argument on $\mathcal{O}_{q^{-1}}\left(M_{n}(\mathbb{C})\right)$ gives that $\eta^{\prime}\left(C\left(\sigma_{1}^{\prime}\right)\right) \subseteq D_{n}^{S_{n-1}}$ where $S_{n-1}$ still acts by permuting $t_{2}, \ldots, t_{n}$. Hence, $\eta\left(C\left(\sigma_{1}\right)\right)$ is symmetric in $t_{1}, \ldots, t_{n-1}$ too so we got that $\eta\left(C\left(\sigma_{1}\right)\right)$ is symmetric in all the variables $t_{1}, \ldots, t_{n}$ by $n \geq 3$.

Remark 5.1. In fact, the proof of injectivity of $\eta$ is valid in the case $n=2$ too, but the symmetry argument used to prove $\eta\left(C\left(\sigma_{1}\right)\right) \subseteq \mathbb{C}\left[t_{1}, \ldots, t_{n}\right]^{S_{n}}$ does not give anything if $n=2$. That is why we had to start the induction at $n=2$ instead of $n=1$.

Remark 5.2. As it is discussed in [DL1], the set of cocommutative elements in $\mathcal{O}_{q}\left(G L_{n}(\mathbb{C})\right)$ is the ring of invariants under the right coaction

$$
\begin{aligned}
\alpha: \mathcal{O}_{q}\left(G L_{n}(\mathbb{C})\right) \rightarrow \mathcal{O}_{q}\left(G L_{n}(\mathbb{C})\right) \otimes \mathcal{O}_{q}\left(G L_{n}(\mathbb{C})\right) \\
a \mapsto \sum a_{(2)} \otimes a_{(3)} S\left(a_{(1)}\right)
\end{aligned}
$$

where we use Sweedler's notation. Although this coaction does not agree with the right adjoint coaction

$$
a \mapsto \sum a_{(2)} \otimes S\left(a_{(1)}\right) a_{(3)}
$$

of the Hopf algebra $\mathcal{O}_{q}\left(G L_{n}(\mathbb{C})\right)$ (that is also mentioned in the referred article) but they differ only by the automorphism $S^{2}$. Hence, by Theorem 1.1, the invariants of the right adjoint coaction also form a maximal commutative subalgebra.

We get other maximal commutative subalgebras by applying automorphisms of the algebras $\mathcal{O}_{q}\left(G L_{n}(\mathbb{C})\right), \mathcal{O}_{q}\left(M_{n}(\mathbb{C})\right)$ or $\mathcal{O}_{q}\left(S L_{n}(\mathbb{C})\right)$, though they do not have many automorphisms: it is proved in $[\mathrm{Y} 2$ establishing a conjecture stated in [LL that the automorphism group of $\mathcal{O}_{q}\left(M_{n}(\mathbb{C})\right)$ is generated by the transpose 
operation on the variables and a torus that acts by rescaling the variables $x_{i, j} \mapsto$ $c_{i} d_{j} x_{i, j}\left(c_{i}, d_{j} \in \mathbb{C}^{\times}\right)$.

\section{REFERENCES}

[AY] A Aizenbud, O Yacobi, A quantum analogue of Kostant's theorem for the general linear group, Journal of Algebra 343 (1), 183-194.

[AZ] V. V. Antonov, A. N. Zubkov, Coinvariants for a coadjoint action of quantum matrices, Algebra and Logic, 48 (2009), no 4, 239.

[B] P. Baumann, Another proof of Joseph and Letzter's separation of variables theorem for quantum groups, Transform. Groups 5 (1) (2000) 3-20.

[BS] J. P. Bell, L. W. Small, Centralizers in domains of Gelfand-Kirillov dimension 2, Bull. Lond. Math. Soc. 36 (2004), no. 6, 779-785.

[BG] K. A. Brown, K. R. Goodearl, Lectures on Algebraic Quantum Groups, Birkhauser (2002).

[DL1] M. Domokos, T. H. Lenagan, Conjugation coinvariants of quantum matrices, Bull. London Math. Soc. 35 (2003) 117-127.

[DL2] M. Domokos, T. H. Lenagan, Representations rings of quantum groups, J. Algebra 282 (2004), 103-128.

[GL] K. R. Goodearl, T. H. Lenagan, Quantum Determinantal Ideal, Duke Mathematical Journal (2000).

[H] T. Hayashi, Quantum deformations of classical groups, Publ. RIMS Kyoto Univ. 28 (1992) $57-81$.

[JL] A. Joseph and G. Letzter, Separation of variables for quantized enveloping algebras, Amer. J. Math. 116 (1994), 127-177.

[LL] S. Launois and T. H. Lenagan, Primitive ideals and automorphisms of quantum matrices, Algebr. Represent. Theory 10 (2007), no. 4, 339-365.

[MNY] K. Mimachi, M. Noumi, H. Yamada, Finite dimensional representations of the quantum group $G L_{q}(N, \mathbb{C})$ and the zonal spherical functions on $\mathcal{U}_{q}(n) / \mathcal{U}_{q}(n-1)$, Japan. J. Math. 19 (1993) 31-80.

[RS] J. Richter, S. Silvestrov, Centralizers in Ore extensions over polynomials rings, Int. Electron. J. Algebra 15 (2014), 196-207.

[Y1] M. Yakimov, On the spectra of quantum groups, Memoirs Amer. Math. Soc. 229 (2014), no. 1078.

[Y2] M. Yakimov, The Launois-Lenagan conjecture, J. Algebra 392 (2013), 1-9. 